\title{
Bone marrow-derived hematopoietic cells generate cardiomyocytes at a low frequency through cell fusion, but not transdifferentiation

\author{
Jens M Nygren ${ }^{1}$, Stefan Jovinge ${ }^{1,2}$, Martin Breitbach ${ }^{3}$, Petter Säwén ${ }^{1}$, Wilhelm Röll ${ }^{4}$, Jürgen Hescheler ${ }^{5}$, \\ Jalal Taneera ${ }^{1}$, Bernd K Fleischmann ${ }^{3}$ \& Sten Eirik W Jacobsen ${ }^{1}$
} \\ Recent studies have suggested that bone marrow cells might possess a much broader differentiation potential than previously appreciated. In most cases, the reported efficiency of such plasticity has been rather low and, at least in some instances, is a consequence of cell fusion. After myocardial infarction, however, bone marrow cells have been suggested to extensively regenerate cardiomyocytes through transdifferentiation. Although bone marrow-derived cells are already being used in clinical trials, the exact identity, longevity and fate of these cells in infarcted myocardium have yet to be investigated in detail. Here we use various approaches to induce acute myocardial injury and deliver transgenically marked bone marrow cells to the injured myocardium. We show that unfractionated bone marrow cells and a purified population of hematopoietic stem and progenitor cells efficiently engraft within the infarcted myocardium. Engraftment was transient, however, and hematopoietic in nature. In contrast, bone marrow-derived cardiomyocytes were observed outside the infarcted myocardium at a low frequency and were derived exclusively through cell fusion.
}

Hematopoietic stem cells (HSCs) residing in adult bone marrow possess the unique ability to self-renew and differentiate into multiple lineages. In humans, HSCs are both necessary and sufficient for the lifelong regeneration of more than 1 million blood cells per second (ref. 1). A number of recent studies have suggested that bone marrow cells or enriched HSCs, upon transplantation into adult recipients, might also transdifferentiate and contribute to the regeneration of a variety of nonhematopoietic lineages in multiple organs ${ }^{2-8}$. These initial findings have provoked extensive follow-up studies; some cast doubt on the biological significance and even the existence of such transdifferentiation $^{9-13}$, and others suggest alternative mechanisms for the observed developmental plasticity of transplanted bone marrow cells ${ }^{14-18}$. In parallel, another line of studies has continued to support and extend the concept of the developmental plasticity of bone marrow-derived cells ${ }^{19-25}$. The reported ability of bone marrow cells to regenerate cardiomyocytes upon transplantation into infarcted myocardium remains to date unsurpassed, not only with regard to the level of potential transdifferentiation observed ${ }^{7}$, but also in that this regeneration has been suggested to result in improved cardiac function ${ }^{26}$. Based on these studies, but without further evaluation of the functionality and durability of bone marrow-derived cardiomyocytes, a number of clinical trials have been initiated worldwide in which patients undergoing acute myocardial infarction have been transplanted with autologous bone marrow cells ${ }^{27-29}$. Using different acute cardiac injury models, including ischemic myocardial infarction, we have evaluated the ability of bone marrow cells to regenerate and sustain reconstitution of cardiomyocytes. Our aim was to establish in detail the extent and durability of the bone marrow-derived reconstitution of cardiomyocytes, and the cellular mechanism responsible for the observed developmental plasticity.

\section{RESULTS}

No mobilization of hematopoietic progenitors following infarction HSCs have recently been shown to migrate continually from bone marrow into peripheral blood, under steady-state conditions ${ }^{30}$. We thus argued that if transplantation of HSC-enriched bone marrow cells can restore cardiac function after infarction ${ }^{7,26}$, myocardial infarction might itself trigger physiological mechanisms that promote hematopoietic stem and progenitor cell mobilization. To investigate this possibility, we examined the peripheral blood of mice which had undergone myocardial infarction for potential increases in hematopoietic stem and progenitor cells (Fig. 1). In agreement with previous studies, steady-state peripheral blood contained low levels of in vivo reconstituting cells and myeloid progenitors, which increased considerably upon in vivo treatment with cytokines (Fig. 1a-c). In contrast, mice which had undergone left coronary artery (LCA) liga-

\footnotetext{
${ }^{1}$ Hematopoietic Stem Cell Laboratory, Lund Strategic Research Center for Stem Cell Biology and Cell Therapy, Lund University, BMC B10, Klinikgatan 26, 22184 Lund, Sweden. ${ }^{2}$ Department of Cardiology, University Hospital MAS, Lund University, 20502 Malmö, Sweden. ${ }^{3}$ Institute of Physiology I, University of Bonn, Argelander-Str. 2a, 53115 Bonn, Germany. ${ }^{4}$ Department of Cardiac Surgery, University of Bonn, Sigmund-Freud-Str. 25, 53105 Bonn, Germany. ${ }^{5}$ Institute of Neurophysiology, University of Cologne, Robert-Koch-Str. 39, 50931 Cologne, Germany. Correspondence should be addressed to S.E.W.J.

(Sten.Jacobsen@stemcell.Iu.se).

Published online 25 April 2004; doi:10.1038/nm1040
} 
ARTICLES

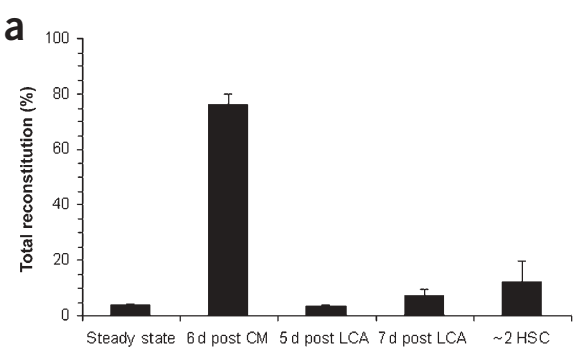

d
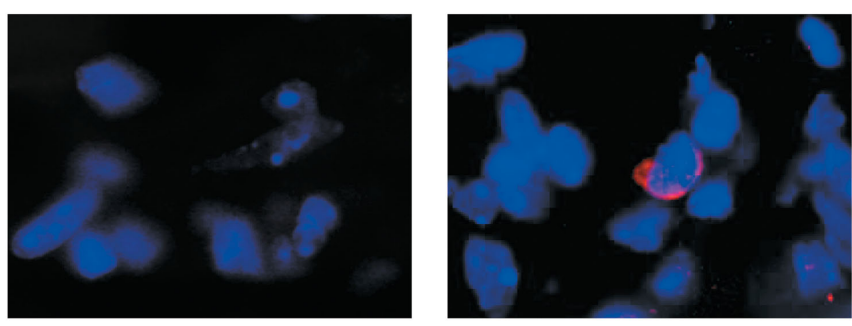

b

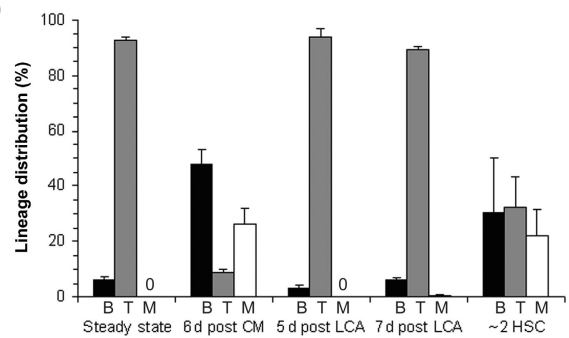

C

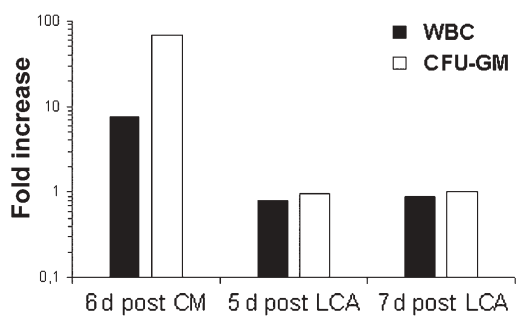

Figure 1 Evaluation of hematopoietic stem and progenitor cell mobilization after myocardial infarction. (a) Contribution of transplanted peripheral blood from steady state $(n=6)$, cytokine-mobilized mice $(\mathrm{CM} ; n=3)$ or LCA-ligated mice ( 5 or $7 \mathrm{~d}$ after ligation; $n=4$ and 3 , respectively) to total peripheral blood cellularity of irradiated recipients $6-8$ weeks after transplantation. As a reference, steady-state peripheral blood was transplanted together with approximately two purified HSCs $(n=2)$. (b) Lineage distribution of reconstituting cells ( $\mathrm{B}, \mathrm{B}$ cell; $\mathrm{T}$, T cell; $\mathrm{M}$, myeloid cell). Contribution to myeloid lineage is a requisite hallmark of HSC reconstitution. (c) Fold increase of peripheral blood white blood cells (WBC) and colony-forming cells (CFU-GM) after cytokine treatment or LCA ligation. Data are from one of three experiments with similar results. Values are presented as mean \pm s.e.m. (d) No c-Kit ${ }^{+}$(red) cells are observed in infarcted myocardium (left) unless mobilized by treatment with cytokines (right). Hoechst nuclear stain is in blue. Magnification, $\times 400$.

tion or sham operation did not show any increase in peripheral blood cellularity or progenitor and stem cell activity, as evaluated in vitro and in vivo (Fig. 1a-c). This failure to detect hematopoietic stem or progenitor cell mobilization after LCA ligation was not caused by trapping of potentially mobilized cells in the infarcted myocardium, as we did not observe any $\mathrm{c}-\mathrm{Kit}^{+}$stem or progenitor cells in the infarcted myocardium unless they were mobilized with cytokine treatment ( $<1$ cell per $8-\mu \mathrm{m}$ section; Fig. 1d). Similarly, analysis of progenitor cell activity (colony-forming cells) and stem or progenitor cell $\left(\mathrm{CD} 34^{+} \mathrm{c}-\mathrm{Kit}^{+}\right)$frequency by FACS did not reveal any trapping of hematopoietic stem or progenitor cells in the spleen after myocardial infarction (data not shown).

\section{Engraftment of bone marrow cells in infarcted myocardium}

Previous studies had suggested that HSC-enriched $\left(\mathrm{Lin}^{-} \mathrm{c}-\mathrm{Kit}^{+}\right)$bone marrow cells efficiently engraft infarcted myocardium, resulting in regeneration of cardiomyocytes as early as $9 \mathrm{~d}$ after infarction ${ }^{7}$, but the durability of this engraftment was not investigated. Ongoing clin- ical trials of bone marrow transplantation into myocardial infarction patients use unfractionated bone marrow cells ${ }^{27-29}$ that include $\mathrm{Lin}^{-} \mathrm{c}-\mathrm{Kit}^{+}$hematopoietic stem and progenitor cells ${ }^{7,31}$. We therefore transplanted $1 \times 10^{6}$ unfractionated bone marrow cells containing a GFP transgene under the control of a $\beta$-actin promoter $^{32}$ (this population contains $\sim 10,000 \mathrm{Lin}^{-} \mathrm{c}-\mathrm{Kit}^{+}$cells and $\sim 100$ HSCs; ref. 33) into infarcted mouse myocardium after LCA ligation (Fig. 2), and investigated the engraftment and fate of those cells. In accordance with Orlic et al. ${ }^{7}$, high levels of $\mathrm{GFP}^{+}$cells were detected within the infarcted myocardium of all mice investigated after 9 d (Fig. 2a,b). However, the engraftment was sustained only at very low levels after $28 \mathrm{~d}$ (Fig. 2c). Staining revealed that all of the engrafted $\mathrm{GFP}^{+}$cells were positive for the pan-hematopoietic cell surface antigen CD45 (Fig. 2b-e) and negative for cardiac-specific $\alpha$-actinin (Fig. 2f-h), troponin $\mathrm{T}$ and connexin-43 (data not shown). At both 9 and $28 \mathrm{~d}$ after infarction, the cells were small and round, a morphology consistent with that of $\mathrm{CD} 45^{+}$hematopoietic cells. We repeated the analysis on hearts of mice subjected to myocardial damage through

Table 1 Engraftment of mobilized GFP+ bone marrow cells into infarcted myocardium

\begin{tabular}{|c|c|c|c|c|c|c|c|c|c|c|c|}
\hline \multirow[b]{3}{*}{ Expt $^{a}$} & \multicolumn{3}{|c|}{ Donor cells } & \multirow[b]{3}{*}{$n^{\mathrm{b}}$} & \multicolumn{7}{|c|}{ Cardiac lesion } \\
\hline & \multirow[b]{2}{*}{ Source } & \multirow[b]{2}{*}{$\begin{array}{l}\text { Transgene } \\
\text { marker }\end{array}$} & \multirow[b]{2}{*}{$\begin{array}{l}\% \text { BM GFP } \\
\text { chimerism }\end{array}$} & & \multirow[b]{2}{*}{ Procedure } & \multirow[b]{2}{*}{$\begin{array}{l}\text { Time of } \\
\text { analysis }\end{array}$} & \multirow[b]{2}{*}{$\begin{array}{l}\% \text { GFP } \\
\text { chimerism }\end{array}$} & \multicolumn{3}{|c|}{$\%$ of $\mathrm{GFP}^{+}$cells } & \multirow[b]{2}{*}{$\begin{array}{l}\times 40 \text { fields } \\
\text { (sections) }^{d}\end{array}$} \\
\hline & & & & & & & & $\begin{array}{l}\alpha \text {-actinin }^{-} \\
\text {CD45 }^{+}\end{array}$ & $\begin{array}{l}\alpha-\text { actinin }^{-} \\
\text {CD45 }^{-}\end{array}$ & $\begin{array}{l}\text { Cardiac } \\
\text { marker } \\
\text { positive }^{c}\end{array}$ & \\
\hline 1 & WBM & $\beta$-GFP & $76.1 \pm 5.5$ & 4 & LCA/Mob & $9 d$ & $48.4 \pm 7.1$ & 99.9 & 0.1 & 0 & 47 (19) \\
\hline 2 & WBM & $\beta$-GFP & $76.1 \pm 5.5$ & 4 & LCA/Mob & $28 d$ & $28.6 \pm 6.1$ & 99.9 & 0.1 & 0 & $58(26)$ \\
\hline 3 & WBM & $\beta$-GFP & $84.5 \pm 4.2$ & 5 & Cryo/Mob & $28 d$ & $36.8 \pm 3.3$ & 99.8 & 0.2 & 0 & $62(25)$ \\
\hline 4 & WBM & $\alpha$-GFP & $79.0 \pm 2.2$ & 2 & LCA/Mob & $28 d$ & 0 & NA & NA & NA & NA (40) \\
\hline 5 & WBM & $\alpha$-GFP & $86.5 \pm 2.3$ & 5 & Cryo/Mob & $28 d$ & 0 & NA & NA & NA & NA (25) \\
\hline 6 & LSKCD45 & $\beta$-GFP & $64.6 \pm 6.2$ & 3 & LCA/Mob & $9 \mathrm{~d}$ & $59.0 \pm 9.5$ & 99.8 & 0.2 & 0 & $10(10)$ \\
\hline 7 & LSKCD45 & $\beta$-GFP & $64.6 \pm 6.2$ & 3 & LCA/Mob & $28 d$ & $18.2 \pm 9.9$ & 99.8 & 0.2 & 0 & $12(12)$ \\
\hline
\end{tabular}

${ }^{a}$ Each experimental approach (combination of transgene, myocardial injury and time of analysis) was repeated at least once with similar results. ${ }^{b}$ Number of mice in each experiment. ${ }^{\mathrm{C}}$ Expression of cardiac markers (cardiac troponin T, $\alpha$-actin, connexin-43 and Nkx-2.5) on GFP+ cells. Specificity of each marker was confirmed using control antibodies and positive and negative control tissues (see Methods). dNumber of $\times 40$ fields (total number of sections screened given in parenthesis) analyzed within the cardiac lesion for GFP chimerism and coexpression of GFP with $\alpha$-actinin and CD45. WBM, whole bone marrow cells; LSKCD45, Lin ${ }^{-} \mathrm{c}-\mathrm{Kit}+\mathrm{Sca}-1^{+} \mathrm{CD} 45^{+}$cells; $\beta$-GFP, GFP under control of $\beta$-actin promoter; $\alpha$-GFP, GFP under control of $\alpha$-actin promoter; LCA/Mob, LCA ligation and cytokine mobilization; Cryo/Mob, cryoinjury and cytokine mobilization; NA, not applicable. Values are presented as mean \pm s.d. 
cryoinjury, and again found high engraftment within the damaged myocardium of $\mathrm{GFP}^{+} \mathrm{CD} 45^{+} \alpha$-actinin ${ }^{-}$cells that declined in numbers between 9 and $28 \mathrm{~d}$ after infarction (data not shown).

Because other types of bone marrow cells might negatively affect the ability of hematopoietic stem and progenitor cells to engraft and transdifferentiate, we next transplanted $2 \times 10^{5}$ purified Lin $^{-} \mathrm{c}-\mathrm{Kit}^{+}$ bone marrow cells into the hearts of LCA-ligated mice and evaluated their engraftment after $9 \mathrm{~d}$ (as in ref. 7). Similar to what was observed after transplantation of whole bone marrow cells, donor $\mathrm{GFP}^{+}$cell engraftment was extensive at the sites of injection (infarction border zone and infarcted myocardium). The engrafted cells proved to be exclusively of the hematopoietic lineage, as they expressed CD45 $(100 \%)$ as well as the specific myeloid blood lineage marker Gr$1 /$ Mac-1 (99\%), but never the cardiac markers $\alpha$-actinin, troponin T, connexin-43 or Nkx-2.5 (Supplementary Table 1 online).

\section{Engraftment in infarcted myocardium after mobilization}

We next applied a noninvasive and highly efficient technique to deliver hematopoietic stem and progenitor cells to the infarcted myocardium. The physiological migration of bone marrow hematopoietic stem and progenitor cells into peripheral blood and peripheral sites ${ }^{30}$ can be markedly enhanced by administering hematopoietic cytokines ${ }^{34}$. This procedure, which has few side effects, is used routinely in the clinic to mobilize stem and progenitor cells to peripheral blood in HSC transplant donors ${ }^{35}$, and recent studies have suggested that the technique might also be an efficient way to repair infarcted myocardium ${ }^{26}$. To specifically investigate the potential contribution of mobilized bone marrow cells to cardiomyocyte regeneration, we first generated mice chimeric for $\mathrm{GFP}^{+}$bone marrow by transplanting lethally irradiated (bone marrow ablated) lacZ-transgenic ${ }^{36}$ Rosa26 mice with whole bone marrow cells or purified $\mathrm{Lin}^{-} \mathrm{c}-\mathrm{Kit}^{+} \mathrm{Sca}-{ }^{+} \mathrm{CD} 45^{+}\left(\mathrm{LSKCD}^{+} 5^{+}\right)$HSCs (ref. 6) from mice transgenic for GFP under the control of a $\beta$-actin promoter ${ }^{32}$. Four weeks after transplantation, blood cells were confirmed to be predominantly derived from transplanted $\mathrm{GFP}^{+}$cells (experiments 1 and 2 in Table 1 ) and contributed to all blood cell lineages (B cells, 49\%; T cells, 16\%; myeloid cells, $22 \%$ ). Six weeks after transplantation, LCA ligation and treatment of the mice with cytokines (Fig. 3a) resulted in high and prolonged levels of hematopoietic stem and progenitor

Figure 2 Direct transplantation and engraftment of $\beta$-actin/GFP-transgenic bone marrow cells into infarcted myocardium. (a) Engraftment of GFP ${ }^{+}$ cells (green, indicated by arrows) predominantly within infarcted myocardium (IM) rather than viable myocardium (VM), $9 \mathrm{~d}$ after infarction and transplantation. (b) $\mathrm{GFP}^{+}$cells in region boxed in a expressed CD45 (red; inset). EP, epicardium; $E N$, endocardium. (c) Reduced engraftment of $\mathrm{GFP}^{+}$cells on day 28; $\mathrm{GFP}^{+}$cells still expressed CD45 (inset). (d-f) Engrafted bone

marrow-derived cells in infarcted myocardium expressed GFP (d) and CD45 (blue; e), but not $\alpha$-actinin (red; $\mathbf{f}$ ). (g) Merger of images in $\mathbf{d}-\mathbf{f}$.

(h) Positive control for $\alpha$-actinin staining in viable myocardium. Panels show representative images from six mice on day 9 and nine mice on day 28 after transplantation. Magnification, $\times 20(a)$, $\times 120(\mathbf{b}, \mathbf{c}), \times 200(\mathbf{d}-\mathbf{h})$ or $\times 500$ (insets in $\mathbf{b}, \mathbf{c}$ ). a

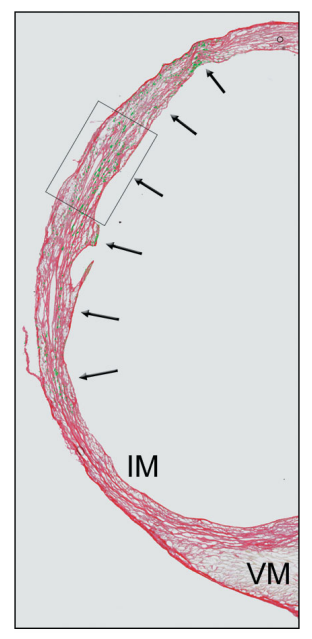

d

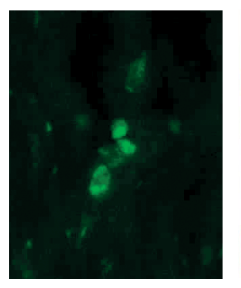

cells in peripheral blood. Nine days after infarction, high levels of $\mathrm{GFP}^{+}$cells were observed within the infarcted myocardium (Fig. 3b and Table 1), whereas very limited engraftment was observed within the uninjured, viable part of the heart and the infarction border zone. $\mathrm{GFP}^{+}$cells within the infarcted myocardium had a morphology resembling that of hematopoietic cells, rather than cardiomyocytes, and $>99 \%$ expressed CD 45 but did not express $\alpha$-actinin, troponin $\mathrm{T}$ or Nkx-2.5 (Fig. 3b and Table 1). In further support of their hematopoietic nature, the engrafting $\mathrm{GFP}^{+} \mathrm{CD} 45^{+}$cells also expressed markers specific for myeloid cells (Gr-1/Mac-1, 80\%), T cells (CD4/CD8, 17\%) or B cells (B220, 5\%; Supplementary Fig. 1 online). The initial engraftment of $\mathrm{GFP}^{+}$cells on day 9 decreased but remained high in the infarcted myocardium on day 28 (Fig. $3 \mathrm{c}$ and Table 1), and virtually all $\mathrm{GFP}^{+}$cells continued to express CD45 (Fig. 3c) but did not express $\alpha$-actinin (Fig. 3d), troponin $\mathrm{T}$ or Nkx-2.5 (Table 1). Similar data were obtained after cryoinduced myocardial injury (Table 1 ).

In four experiments $(n=21)$, we treated mice with cytokines before, rather than only after, infarction. In these experiments, cytokine mobilization of mice transplanted with whole bone marrow was initiated $2 \mathrm{~d}$ before LCA ligation and continued for $5 \mathrm{~d}$ afterward. The results were similar to those obtained when mobilizing only after the infarction (data not shown).

The typical difference in morphology between engrafted $\mathrm{GFP}^{+}$cells and native cardiomyocytes was corroborated at the single-cell level after dissociation with Langendorf perfusion on day 28 (Fig. 3e). $\mathrm{GFP}^{+}$cells were small and round, whereas native cardiomyocytes had a typical rod shape and cross-striation.

Further evidence that mobilized bone marrow-derived cells could not adapt a cardiomyocyte fate was obtained by transplanting lethally irradiated wild-type mice with bone marrow cells from mice expressing GFP under the control of the muscle-specific $\alpha$-actin

e

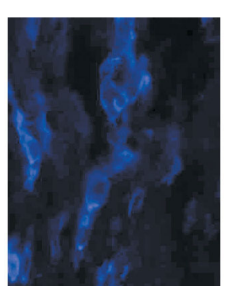

b

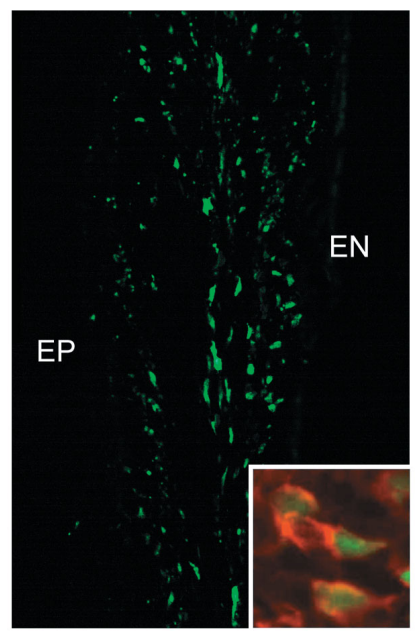

f

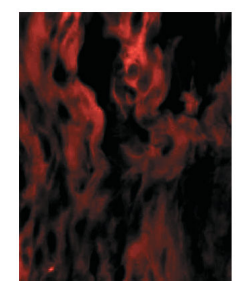

C

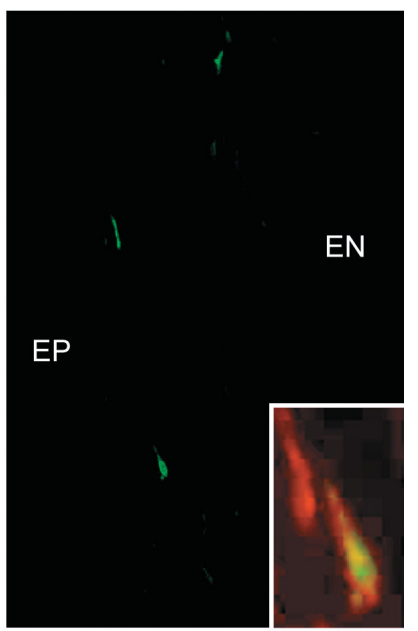

g

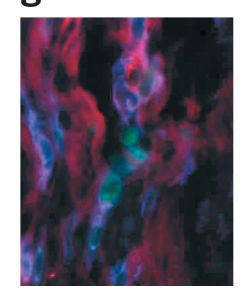

h

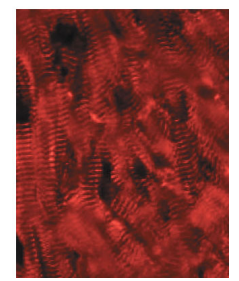


promoter ${ }^{37}$. After myocardial infarction and cytokine-induced mobilization, we examined the hearts of recipients for potential regeneration with $\mathrm{GFP}^{+}$cardiomyocytes. In a total of 54 sections analyzed from seven hearts, we observed no cells within the infarcted myocardium that had the high GFP expression levels observed in all cardiomyocytes from $\alpha$-actin/GFP-transgenic mice (Table 1 and Supplementary Fig. 2 online). However, large numbers of small, round $\mathrm{GFP}^{\mathrm{lo}} \mathrm{CD} 45^{+}$cells were observed in the infarcted myocardium, indicating engraftment of the mobilized $\alpha$-actin/GFPtransgenic bone marrow cells. The low levels of GFP observed in these $\mathrm{CD} 45^{+}$cells (most likely caused by promoter leakiness) were comparable to those observed in the peripheral blood and bone marrow of the $\alpha$-actin/GFP-transgenic mice.

\section{Fusion of bone marrow-derived cells with cardiomyocytes}

Cytokine-mobilized bone marrow cells (derived from GFPtransgenic whole bone marrow or purified LSKCD $45^{+}$cells) engrafting within the myocardial infarction did not acquire a cardiomyocyte morphology or phenotype. However, a low frequency of distinct

a

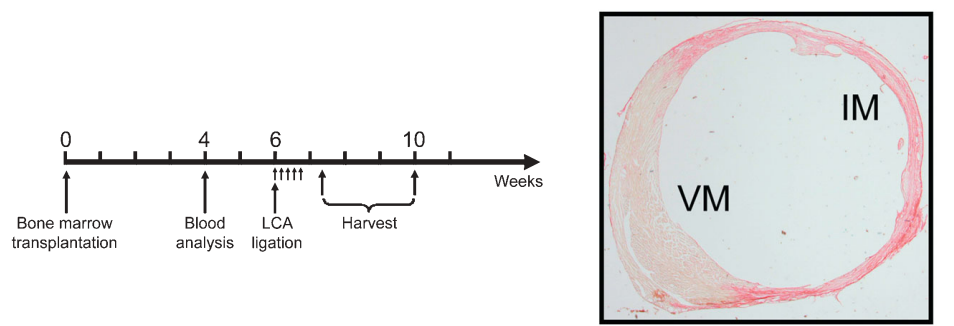

b
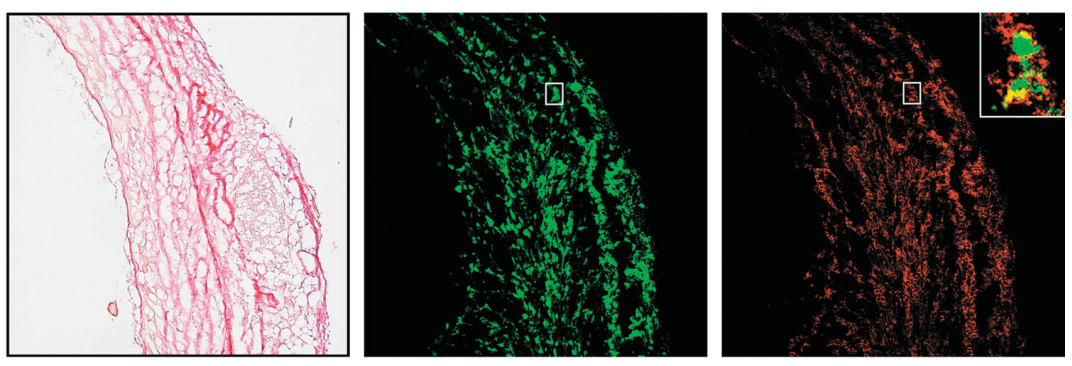

C
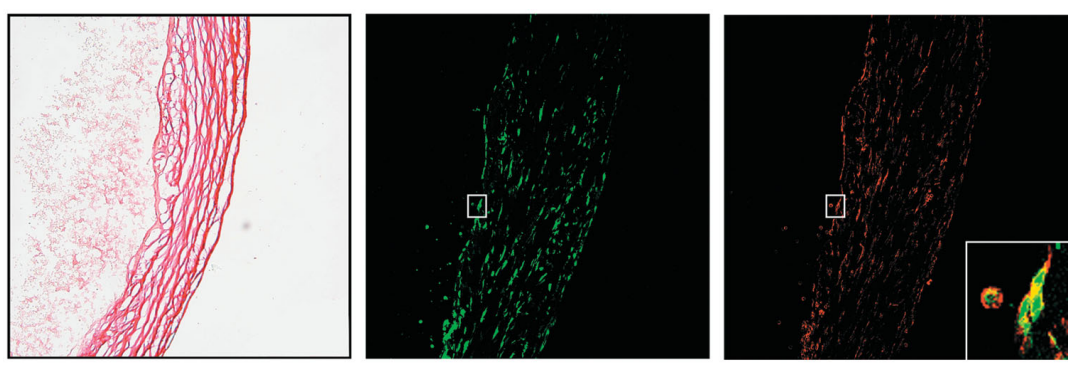

d
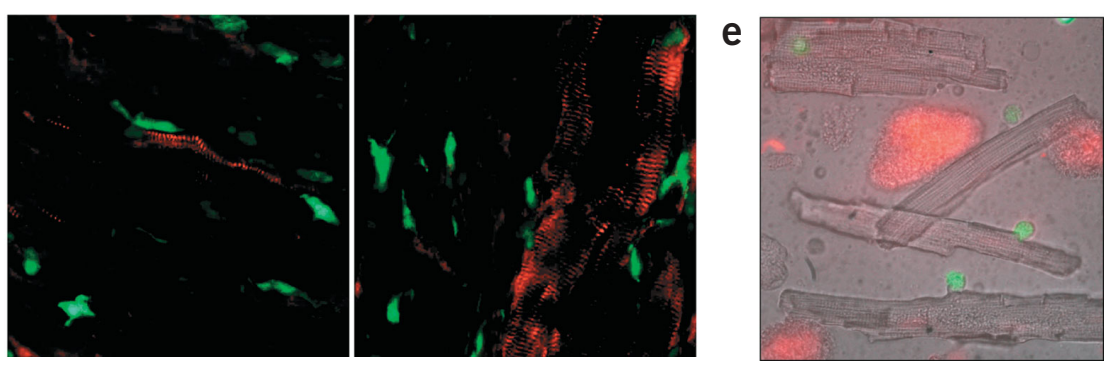

$\mathrm{GFP}^{+}$cells with characteristic cardiomyocyte morphology, including cross-striation, was observed outside or in the border zone of the myocardial infarction. GFP expression by these cells was confirmed by staining with GFP-specific antibodies (Fig. 4a), and their cardiomyocyte identity was further confirmed by their expression of $\alpha$-actinin (Fig. 4b), $\alpha$-actin (Fig. 4c), cardiac troponin T (Fig. 4d) and connexin-43 (Fig. 4e), as well as their lack of expression of CD45 (Fig. 4f). Although they were rare, multiple $\mathrm{GFP}^{+}$cardiomyocytes were identified in all infarcted mice analyzed on day 28 , representing $0.75 \%$ of all $\mathrm{GFP}^{+}$cells and $0.0065 \%$ of all cardiomyocytes in mice reconstituted with whole bone marrow cells. Similar frequencies were observed in the bone marrow of mice reconstituted with whole bone marrow cells and purified LSKCD $45^{+}$cells.

Because the mobilized bone marrow cells were $\mathrm{GFP}^{+}$and the endogenous cardiomyocytes carried the lac $Z$ transgene, we were able to address whether $\mathrm{GFP}^{+}$cardiomyocytes were derived through cell fusion rather than transdifferentiation. Notably, whereas control X-gal staining of cardiomyocytes from $\beta$-actin/GFP-transgenic donor mice was negative, all $\mathrm{GFP}^{+}$cardiomyocytes in the infarcted Rosa26 mice, which were reconstituted with whole bone marrow cells or purified LSKCD $45^{+}$cells, were positive for X-gal staining (Fig. 5a-d). Confocal microscopy detected the $\mathrm{X}$-gal precipitate within the cytoplasm of the $\mathrm{GFP}^{+}$cardiomyocytes (Fig. $5 \mathrm{e}$ and Supplementary Fig. 3 online), indicating conclusively that they were generated through fusion rather than transdifferentiation. In addition, bone marrow-derived cardiomyocytes were typically binucleated when stained with Hoechst 33342 (Fig. 5f). In contrast to bone marrow-reconstituted mice, we did not observe any bone marrow-derived cardiomyocytes in mice in which whole bone marrow cells or $\mathrm{Lin}^{-} \mathrm{C}-$ $\mathrm{Kit}^{+}$cells were transplanted directly into the infarcted myocardium (J.M.N., unpublished observations).

Figure 3 Engraftment of cytokine-mobilized $\beta$-actin/GFP-transgenic bone marrow cells in infarcted myocardium. (a) Left, experimental design. Small arrows indicate cytokine injections. Right, typical heart $9 \mathrm{~d}$ after LCA ligation, stained with picric acid and Sirius red to assess collagen content (red) in injured (IM) and viable (VM) myocardium. (b) Infarcted ventricular myocardium after $9 \mathrm{~d}$ (left), with engrafting $\mathrm{GFP}^{+}$cells (green; middle) expressing CD45 (red; right). Inset shows merger of middle and right images of boxed region. (c) Reduced but sustained engraftment of $\mathrm{GFP}^{+} \mathrm{CD} 45^{+}$cells after $28 \mathrm{~d}$, analyzed as in b. (d) Expression of $\alpha$-actinin (red) in surviving endogenous (GFP-) cardiomyocytes but not in bone marrow-derived $\left(\mathrm{GFP}^{+}\right)$cells in infarcted myocardium (left) and infarct border zone (right). (e) Isolated cells obtained by Langendorf perfusion $28 \mathrm{~d}$ after infarction. Viability of dissociated cells was confirmed by propidium iodide (red) staining. Magnification, $\times 50$ (b and c, left), $\times 80$ (b and c, middle and right), $\times 200$ (d), $\times 320$ (e) or $\times 400$ (insets in $\mathbf{b}, \mathbf{c}$ ). 
Figure 4 Bone marrow-derived GFP ${ }^{+}$ cardiomyocytes in viable myocardium. Data were obtained $28 \mathrm{~d}$ after LCA ligation and cytokine mobilization of Rosa26 mice reconstituted with whole bone marrow cells transgenic for $\beta$-actin/GFP. (a) Candidate cardiomyocyte stained positively with GFPspecific antibodies (red) and exhibited GFP fluorescence (green). (b-e) Cardiomyocytes in viable myocardium were positive for $\alpha$-actinin (red; b), $\alpha$-actin (red; c), cardiac troponin T

(red; d), and gap junction protein connexin-43 (red; e; indicated by arrowheads). Right panels in b-e show merger with GFP fluorescence (green). (f) No expression of panhematopoietic marker CD45 (blue) was observed on $\mathrm{GFP}^{+}$ cardiomyocytes (green). Arrow indicates donorderived $\left(\mathrm{GFP}^{+}\right) \mathrm{CD}^{+} 5^{+}$hematopoietic cell; arrowheads indicate endogenous (GFP ${ }^{-}$) $\mathrm{CD}_{4} 5^{+}$ cells along the $\mathrm{GFP}^{+}$cardiomyocyte.

Magnification is $\times 160$ in all panels. Images were acquired using confocal microscopy.

\section{DISCUSSION}

Our studies confirmed the ability of bone marrow-derived hematopoietic cells to engraft when transplanted directly into the hearts of mice subjected to acute myocardial infarction. In agreement with Orlic et al. ${ }^{7}$, we

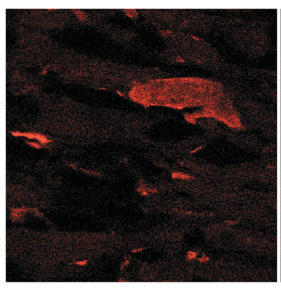

C

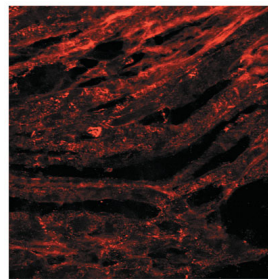

e

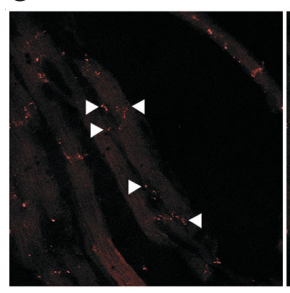

b
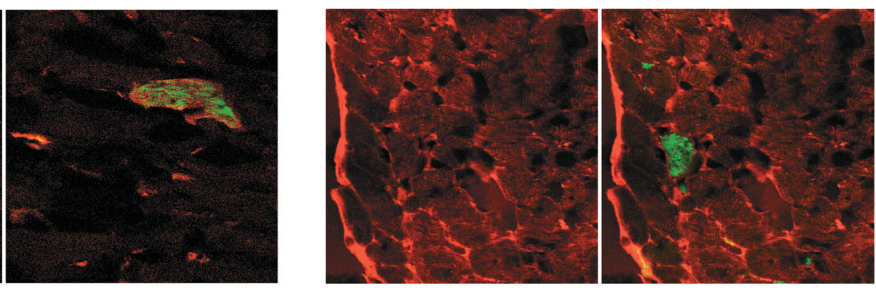

d
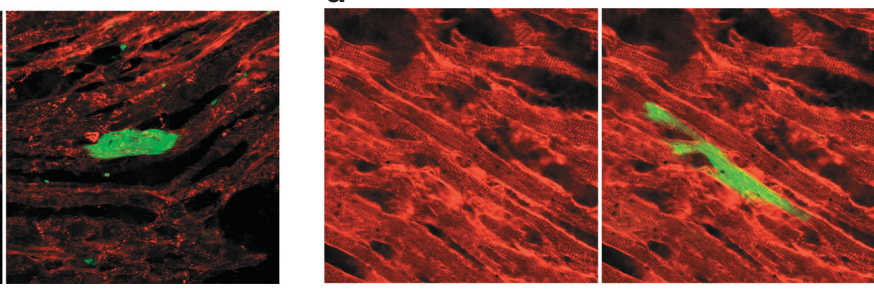

f
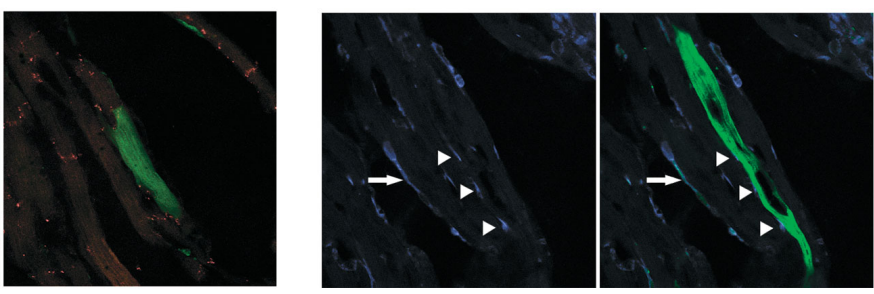
found that the engraftment was highly selective for the damaged myocardium. But whereas Orlic et al. investigated reconstitution only $9 \mathrm{~d}$ after transplantation ${ }^{7}$, we observed that most of the engraftment within the myocardial infarction was lost by day 28. Unexpectedly, both 9 and $28 \mathrm{~d}$ after transplantation, all engrafted bone marrow-derived cells within the injured myocardium, whether they were derived from whole bone marrow or purified Lin $^{-} \mathrm{c}-\mathrm{Kit}^{+}$cells, expressed the pan-hematopoietic marker CD45, coexpressed myeloid blood lineage markers (Gr-1/Mac-1), failed to express cardiac-specific markers, and displayed a cell mor-

phology typical of blood cells rather than cardiomyocytes. These data show that hematopoietic cells, rather than cardiomyocytes, accounted for the transient engraftment observed after direct transplantation of bone marrow-derived cells into infarcted myocardium. The data are in agreement with our earlier findings of scar formation in mice ${ }^{38}$, which points to an invasion by inflammatory cells and their disappearance toward the end of this process.

We also investigated the potential of bone marrow cells to engraft as cardiomyocytes after cytokine mobilization of GFP-transgenic bone a

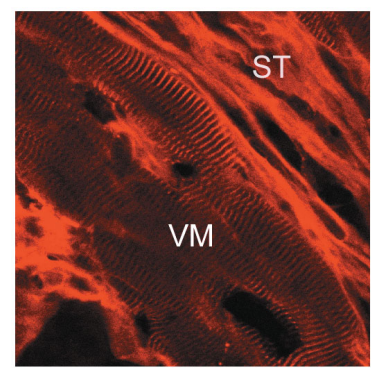

d

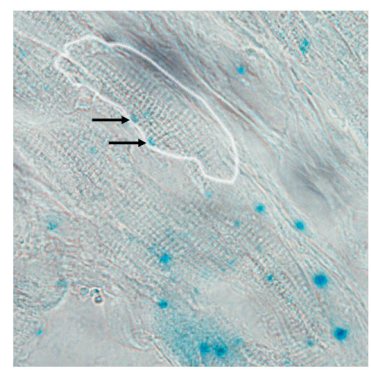

b

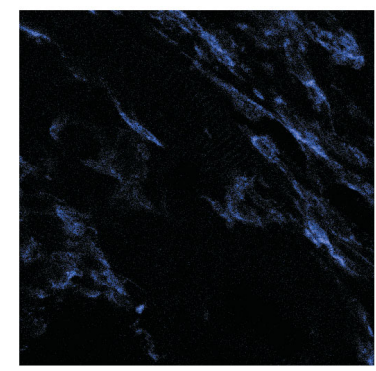

e

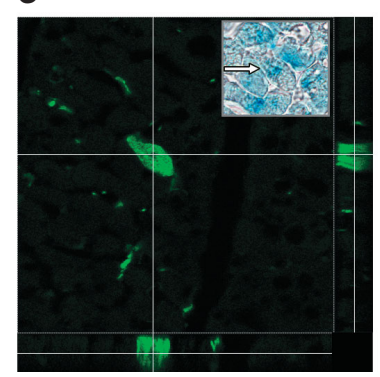

C

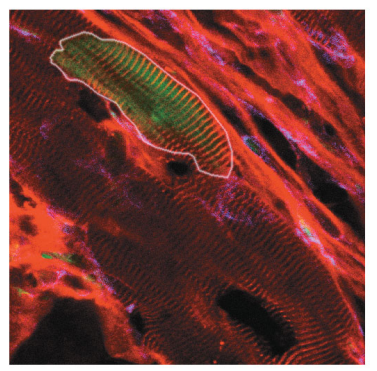

f

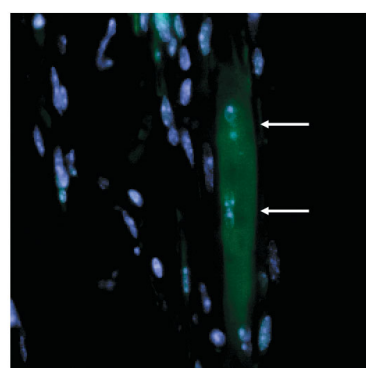

Figure 5 Fusion of bone marrow-derived cells with cardiomyocytes in viable myocardium, $28 \mathrm{~d}$ after LCA ligation and cytokine mobilization of Rosa26 (lacZ-transgenic) mice reconstituted with whole $\beta$-actin/GFP-transgenic bone marrow cells. (a) $\alpha$-actinin-positive cardiomyocytes (red) in the infarct border zone and viable myocardium (VM). Strong but unspecific staining was observed within the scar tissue (ST). (b) CD45+ (blue) cells in scar tissue. (c,d) Cardiomyocyte in viable myocardium, positive for GFP (c; green; merger of $\mathbf{a}$ and $\mathbf{b}$ ) and X-gal (d). X-gal staining of Rosa26 cardiomyocytes gives strong blue punctuate staining in addition to weaker blue staining of the whole muscle fiber. (e) Confocal three-dimensional view showing $X$-gal precipitate (arrow, inset) surrounded by GFP ${ }^{+}$cytoplasm in all dimensions (see also Supplementary Fig. 3 online). (f) $\mathrm{GFP}^{+}$cardiomyocytes in longitudinal sections were binucleated when stained with Hoechst 33342 (blue). Magnification, $\times 160$ $(\mathbf{a}-\mathbf{e})$ or $\times 200(\mathbf{f})$. Images were acquired using confocal (a-c,e), transmission light (d) or fluorescence (f) microscopy. 
marrow cells (derived from whole bone marrow cells or purified HSCs) into peripheral blood and the infarcted myocardium. Although cytokine treatment resulted in higher and more sustained levels of engraftment of bone marrow-derived cells in the infarcted myocardium, the engrafted cells, like those observed after direct injection, retained their hematopoietic identity and no evidence of bone marrow-derived cardiomyocytes was observed within the infarcted myocardium. These data suggest that the enhanced survival and improved cardiac function observed after bone marrow mobilization of infarcted mice ${ }^{26}$ is not likely to be mediated by transdifferentiation of bone marrow cells into cardiomyocytes.

In contrast to what we observed within the infarcted myocardium, after cytokine mobilization we consistently observed a very low number of bone marrow-derived $\mathrm{GFP}^{+}$cardiomyocytes outside the infarction area. These cells had a morphology typical of cardiomyocytes, expressed a number of cardiomyocyte-specific markers and lacked expression of CD45, strongly supporting their identity as cardiomyocytes. Using different transgenic marker genes in the transplanted bone marrow cells and in the infarcted myocardium, we showed that these cardiomyocytes were derived not from transdifferentiation of bone marrow cells, as had been suggested in previous studies using ischemic and non-ischemic cardiac injury models ${ }^{8,39}$, but from cell fusion. Our findings that bone marrow-derived cardiomyocytes were observed only at a low frequency and were generated through cell fusion provides an explanation for why we and others ${ }^{8}$ had not observed bone marrow-derived cardiomyocytes within the infarcted myocardium, where few or no intact cardiomyocytes are available as targets for fusion. Notably, whereas bone marrow-derived cardiomyocytes were observed in mice whose bone marrow had been reconstituted with whole bone marrow cells or purified HSCs before infarction, they were not observed in mice in which bone marrow cells were transplanted directly into the infarcted myocardium. This difference could be explained by the delivery of greater numbers of bone marrow cells to the infarcted myocardium in the reconstituted mice, or by more sustained or optimal delivery of these cells. It could also, however, be a consequence of the bone marrow transplantation procedure or the conditioning procedure used before transplantation.

A low frequency of cell fusion in vivo has also been reported between bone marrow cells and hepatocytes ${ }^{16,17}$, suggesting that cell fusion events involving bone marrow-derived cells might occur commonly after different types of tissue damage. Recent studies suggest that bone marrow-derived cells can fuse with cardiomyocytes ${ }^{18}$ in noninjury models, and that progenitors isolated from the myocardium can generate new cardiomyocytes through cell differentiation as well as fusion ${ }^{40}$. But considering the low frequency of these events, in those studies and ours, and the apparent inability of cardiomyocytes generated by cell fusion to expand-solitary fused cardiomyocytes were typically observed in our studies (J.M.N., unpublished observations) —-we feel that it is unlikely that generation of cardiomyocytes by cell fusion could significantly improve cardiac function after myocardial infarction. However, the functional consequences (positive or negative) of bone marrow-derived cardiomyocyte fusion need to be investigated in more detail, particularly in light of recent reports of adverse effects after bone marrow transplantation of myocardial infarction patients ${ }^{41,42}$.

Our studies focused on the reported ability of hematopoietic stem or progenitor cells to form cardiomyocytes through transdifferentiation, as this ability is the experimental basis for ongoing clinical bone marrow transplantation trials in myocardial infarction patients. Although beyond the scope of our studies, bone marrow transplantation could potentially contribute to endogenous cardiomyocyte repair through other mechanisms. Although inspection of the infarcted hearts did not reveal signs of substantial endogenous repair after transplantation of bone marrow-derived cells (J.M.N., unpublished observations), the well-known variation in infarct size in rodents after LCA occlusion would not have allowed us to detect limited endogenous repair. Clearly, other models will be required to carefully address the role of endogenous repair after bone marrow transplantation.

Because bone marrow-derived HSCs continually migrate into peripheral blood, and because this process can be enhanced by soluble factors ${ }^{34}$, it would seem natural that such signals would be upregulated in response to acute tissue damage if hematopoietic cells had a role in the normal repair process. Although we used highly sensitive methods, such as competitive in vivo reconstitution assays, in vitro colonyforming assays and FACS analysis, to detect low levels of mobilized hematopoietic stem and progenitor cells, we did not observe any hematopoietic stem-progenitor cell mobilization in response to acute myocardial infarction. We also found no evidence for trapping of stem and progenitor cells in the damaged myocardium or spleen of infarcted mice. However, because previous studies had implicated bone marrow-derived hematopoietic stem or progenitor cells as a source of transdifferentiated cardiomyocytes ${ }^{7,8}$, we investigated infarcted mice only for potential mobilization of progenitors of the hematopoietic lineage. Thus, ischemic myocardial injury might elicit signals that mobilize nonhematopoietic progenitors from the bone marrow-particularly endothelial progenitors, as previously shown ${ }^{43}$.

Our results are in agreement with two recent reports (published online while our manuscript was under review) that transplanted hematopoietic cells are unable to transdifferentiate into cardiomyocytes after myocardial infarction ${ }^{44,45}$. Our studies extend these findings in several ways. First, in addition to using purified hematopoietic stem cells, we also transplanted unfractionated bone marrow-derived cells into the infarcted myocardium, similar to the procedure used in ongoing clinical trials ${ }^{27-29}$. We found no evidence for the generation of cardiomyocytes in the infarcted region of the myocardium with either type of cell population. Second, we extended the observation that bone marrow-derived cardiomyocytes can be found at low frequency outside the infarcted myocardium ${ }^{45}$ by demonstrating that such cardiomyocytes are generated through cell fusion rather than transdifferentiation. Finally, we showed that the reported improvement in cardiac function after cytokine mobilization ${ }^{7}$ is unlikely to be the result of generation of bone marrow-derived cardiomyocytes.

In conclusion, although high levels of engraftment of hematopoietic cells were achieved in the ischemic myocardium, this engraftment was transient and entirely hematopoietic, and no bone marrow-derived cardiomyocytes were observed in the damaged myocardium. In contrast, very low levels of cardiomyocytes derived from fusion with bone marrow-derived cells were observed outside the infarction area. Our findings challenge some of the experimental bases for the ongoing extensive clinical bone marrow transplantation trials of myocardial infarction patients ${ }^{27-29}$, as these trials were initiated largely on the assumption that bone marrow-derived cells generate high levels of cardiomyocytes through transdifferentiation ${ }^{7,26}$.

\section{METHODS}

Mice. We used mice transgenic for GFP (driven by the $\beta$-actin ${ }^{32}$ or $\alpha$-actin ${ }^{37}$ promoters) and lacZ (Rosa26; ref. 36) on a $\mathrm{C} 57 \mathrm{Bl} / 6$ background. All mice were given sterile food and autoclaved acidified water, and housed under pathogenfree conditions. All experiments were approved by the ethical committees at Lund and Cologne Universities.

Bone marrow and $\mathrm{HSC}$ isolation. Bone marrow cells were isolated, and $\mathrm{Lin}^{-} \mathrm{c}-$ $\mathrm{Kit}^{+}$and $\mathrm{Lin}^{-} \mathrm{c}-\mathrm{Kit}^{+} \mathrm{Sca}-{ }^{+} \mathrm{CD} 34^{-}$HSCs were purified as previously described ${ }^{31}$. In some experiments, $\mathrm{Lin}^{-} \mathrm{c}-\mathrm{Kit}^{+} \mathrm{Sca}-1^{+} \mathrm{CD} 45^{+} \mathrm{HSCs}^{6}$ were sorted 
to show that the cardiomyocytes derived through fusion with bone marrow-derived cells were of hematopoietic $\left(\mathrm{CD} 45^{+}\right)$origin.

Surgical procedures. Surgical procedures were performed as previously described ${ }^{46}$. Myocardial infarctions were generated by ligation of the LCA or through cryoinduced injury. Control (sham-operated) mice underwent the same surgical procedure, but without injury induction.

Phenotypic and morphological evaluation. Hearts were subjected to Langendorf dissociation or in situ perfusion, post-fixation at $4{ }^{\circ} \mathrm{C}$ with Stefanini solution, equilibration in $20 \%$ sucrose and cryosectioning $(8-30 \mu \mathrm{m})$. Sections were permeabilized and stained with $\mathrm{X}^{-g a l}{ }^{8}$ (Rosa26 recipients) and/or stained with primary antibodies to CD45 (Neomarkers), c-Kit (Santa Cruz Biotechnology), $\alpha$-actinin (Sigma), $\alpha$-actin (Sigma), cardiac troponin T (Neomarkers), connexin-43 (BioTrend), Nkx-2.5 (Santa Cruz Biotechnology), hematopoietic lineage markers (CD4, CD8, B220, Gr-1 and Mac-1; all from PharMingen) and GFP (Chemicon). Primary antibodies were visualized with secondary antibodies conjugated to Cy3 and Cy5 (Jackson ImmunoResearch Laboratories), Alexa Fluor 555 (Molecular Probes) or Texas red (Jackson ImmunoResearch Laboratories). Finally, sections were stained with Hoechst 33342 (Sigma). For picric acid and Sirius red staining, sections were incubated with $0.1 \%$ Direct Red 80 (Sigma) in aqueous picric acid (Sigma; pH 2.0), washed in $0.01 \mathrm{M} \mathrm{HCl}$ and dehydrated before mounting. Staining with control antibodies, as well as staining of negative and positive control tissues, was done to verify the specificity of all antibodies and the $\mathrm{X}$-gal staining. Cardiac-specific antibodies were titrated on viable myocardium from untreated adult mice, with the exception of Nkx-2.5, which was titrated on heart tissue from embryonic day 9.5. Hematopoietic marker-specific antibodies were titrated on samples from bone marrow and spleen. Images were taken using fluorescence (Olympus) or confocal (Leica) microscopy. Frequency of donor-derived cardiomyocytes was determined as previously described ${ }^{8}$. All data were independently confirmed by at least two investigators, and investigators from both the Lund and Cologne laboratories confirmed key data independently.

Mobilization of hematopoietic progenitors after myocardial infarction. For evaluation of potential HSC mobilization from bone marrow to peripheral blood in response to myocardial infarction, peripheral blood cells were collected from $\mathrm{C} 57 \mathrm{Bl} / 6$ mice $\left(\mathrm{CD} 45.2^{+}\right)$in steady state, 5 and $7 \mathrm{~d}$ after LCA ligation and $6 \mathrm{~d}$ after cytokine mobilization (see below). For all groups, $250 \mu \mathrm{l}$ peripheral blood cells were transplanted into each lethally irradiated (925 rad) $\mathrm{C} 57 \mathrm{Bl} / 6$ congenic $\left(\mathrm{CD} 45.1^{+} \mathrm{CD} 45.2^{+}\right)$recipient, together with 150,000-200,000 unfractionated bone marrow competitor cells $\left(\mathrm{CD} 45.1^{+}\right)$. For each donor, peripheral blood was transplanted into one recipient. The activity of 20 purified $\mathrm{Lin}^{-} \mathrm{c}-\mathrm{Kit}^{+} \mathrm{Sca}-1^{+} \mathrm{CD} 34^{-}$bone marrow cells ${ }^{33}$, containing approximately two HSCs, transplanted together with $250 \mu$ l of steady-state peripheral blood, was used as a reference. Hematopoietic reconstitution and blood cell lineage distribution was evaluated in peripheral blood 3 and 6-8 weeks after transplantation by flow cytometry (FACSCalibur, Becton Dickinson) of donor-derived (CD45.2 $2^{+}$and competitor-derived (CD45.1 $\left.1^{+}\right)$ reconstitution, as previously described ${ }^{31}$. Peripheral blood cellularity was measured on a Sysmex Kx 21N (Sysmex), and peripheral blood progenitor colony-forming activity (colony-forming unit granulocyte/macrophage) was determined using semisolid clonogenic assays ${ }^{47}$. Only colonies with $>50$ cells were scored.

Bone marrow transplantation of infarcted mice. Myocardial injuries were induced through LCA ligation or cryoinjury as described above. GFP ${ }^{+}$whole bone marrow cells $\left(1.0 \times 10^{6}\right)$ or Lin $^{-}$c-Kit ${ }^{+}$cells $\left(2 \times 10^{5}\right)$ were transplanted, through two $2.5-\mu$ linjections, into the injured myocardium and the bordering viable myocardium immediately after injury induction. The infarcted area was identified by the abrupt change in color. Hearts were collected after 9 or $28 \mathrm{~d}$.

Generation of bone marrow-chimeric mice. Whole bone marrow cells $\left(3 \times 10^{6}\right)$ or $\mathrm{Lin}^{-} \mathrm{c}-\mathrm{Kit}^{+} \mathrm{Sca}-1^{+} \mathrm{CD} 45^{+}$cells $(3,000)$ from GFP-transgenic (under control of $\beta$-actin or $\alpha$-actin promoter) mice were transplanted into lethally irradiated $(925 \mathrm{rad}$ ) wild-type C57Bl/6 or Rosa26 recipients. Hematopoietic reconstitution was evaluated in peripheral blood 4-6 weeks after transplantation as described above.
Cytokine mobilization after myocardial injury. Wild-type or Rosa26 mice with $\mathrm{GFP}^{+}$multilineage hematopoietic chimerism $>60 \%$ underwent myocardial injury, as described above. Bone marrow cells were mobilized to the peripheral blood by five daily injections (starting $1 \mathrm{~h}$ after or $2 \mathrm{~d}$ before injury induction; $5 \mu \mathrm{g} / \mathrm{mouse} / \mathrm{d}$ ) of recombinant human Flt-3 ligand and recombinant mouse granulocyte/macrophage colony-stimulating factor (gifts of Immunex). Hearts were collected after 9 or $28 \mathrm{~d}$.

Note: Supplementary information is available on the Nature Medicine website.

\section{ACKNOWLEDGMENTS}

We thank A. Björklund for fruitful discussions and expert advice on confocal microscopy data; L. Wittman, E. Chodkiewicz, Y. Duan and Z. Wu for expert technical assistance; and the FACS Facility at Lund Strategic Research Center for Stem Cell Biology and Cell Therapy for assistance with cell sorting. This work was supported by the Swedish Heart Lung Foundation, the Juvenile Diabetes Research Foundation, the Swedish Diabetes Foundation, the Swedish Research Council, the Deutsche Forschungsgemeinschaft (FL 276/4-2) and the scientific exchange program North Rhine Westphalia-Sweden. The Lund Stem Cell Center is supported by a Center of Excellence grant in life sciences from the Swedish Foundation for Strategic Research.

\section{COMPETING INTERESTS STATEMENT}

The authors declare that they have no competing financial interests.

Received 30 January; accepted 1 April 2004

Published online at http://www.nature.com/naturemedicine/

1. Lagasse, E., Shizuru, J.A., Uchida, N., Tsukamoto, A. \& Weissman, I.L. Toward regenerative medicine. Immunity 14, 425-436 (2001).

2. Gussoni, E. et al. Dystrophin expression in the $\mathrm{mdx}$ mouse restored by stem cell transplantation. Nature 401, 390-394 (1999).

3. Petersen, B.E. et al. Bone marrow as a potential source of hepatic oval cells. Science 284, 1168-1170 (1999).

4. Mezey, E., Chandross, K.J., Harta, G., Maki, R.A. \& McKercher, S.R. Turning blood into brain: cells bearing neuronal antigens generated in vivo from bone marrow. Science 290, 1779-1782 (2000).

5. Brazelton, T.R., Rossi, F.M., Keshet, G.I. \& Blau, H.M. From marrow to brain: expression of neuronal phenotypes in adult mice. Science 290, 1775-1779 (2000).

6. Lagasse, E. et al. Purified hematopoietic stem cells can differentiate into hepatocytes in vivo. Nat. Med. 6, 1229-1234 (2000).

7. Orlic, D. et al. Bone marrow cells regenerate infarcted myocardium. Nature 410, 701-705 (2001).

8. Jackson, K.A. et al. Regeneration of ischemic cardiac muscle and vascular endothelium by adult stem cells. J. Clin. Invest. 107, 1395-1402 (2001).

9. Kawada, H. \& Ogawa, M. Bone marrow origin of hematopoietic progenitors and stem cells in murine muscle. Blood 98, 2008-2013 (2001).

10. Wagers, A.J., Sherwood, R.I., Christensen, J.L. \& Weissman, I.L. Little evidence for developmental plasticity of adult hematopoietic stem cells. Science 297 2256-2259 (2002)

11. Castro, R.F. et al. Failure of bone marrow cells to transdifferentiate into neural cells in vivo. Science 297, 1299 (2002).

12. McKinney-Freeman, S.L. et al. Muscle-derived hematopoietic stem cells are hematopoietic in origin. Proc. NatI. Acad. Sci. USA 99, 1341-1346 (2002).

13. Geiger, H. et al. Analysis of the hematopoietic potential of muscle-derived cells in mice. Blood 100, 721-723 (2002).

14. Ying, Q.L., Nichols, J., Evans, E.P. \& Smith, A.G. Changing potency by spontaneous fusion. Nature 416, 545-548 (2002).

15. Terada, N. et al. Bone marrow cells adopt the phenotype of other cells by spontaneous cell fusion. Nature 416, 542-545 (2002).

16. Wang, X. et al. Cell fusion is the principal source of bone-marrow-derived hepatocytes. Nature 422, 897-901 (2003).

17. Vassilopoulos, G., Wang, P.R. \& Russell, D.W. Transplanted bone marrow regenerates liver by cell fusion. Nature 422, 901-904 (2003).

18. Alvarez-Dolado, M. et al. Fusion of bone-marrow-derived cells with Purkinje neurons, cardiomyocytes and hepatocytes. Nature 425, 968-973 (2003).

19. Priller, J. et al. Neogenesis of cerebellar Purkinje neurons from gene-marked bone marrow cells in vivo. J. Cell Biol. 155, 733-738 (2001).

20. Krause, D.S. et al. Multi-organ, multi-lineage engraftment by a single bone marrowderived stem cell. Cell 105, 369-377 (2001).

21. Wang, $X$. et al. Kinetics of liver repopulation after bone marrow transplantation. Am. J. Pathol. 161, 565-574 (2002).

22. Quaini, F. et al. Chimerism of the transplanted heart. N. Engl. J. Med. 346, 5-15 (2002).

23. Laflamme, M.A., Myerson, D., Saffitz, J.E. \& Murry, C.E. Evidence for cardiomyocyte repopulation by extracardiac progenitors in transplanted human hearts. Circ. Res. 90, 634-640 (2002).

24. LaBarge, M.A. \& Blau, H.M. Biological progression from adult bone marrow to 
mononucleate muscle stem cell to multinucleate muscle fiber in response to injury. Cell 111, 589-601 (2002).

25. Ianus, A., Holz, G.G., Theise, N.D. \& Hussain, M.A. In vivo derivation of glucosecompetent pancreatic endocrine cells from bone marrow without evidence of cell fusion. J. Clin. Invest. 111, 843-850 (2003).

26. Orlic, D. et al. Mobilized bone marrow cells repair the infarcted heart, improving function and survival. Proc. Natl. Acad. Sci. USA 98, 10344-10349 (2001).

27. Strauer, B.E. et al. Repair of infarcted myocardium by autologous intracoronary mononuclear bone marrow cell transplantation in humans. Circulation 106, 1913-1918 (2002).

28. Assmus, B. et al. Transplantation of progenitor cells and regeneration enhancement in acute myocardial infarction (TOPCARE-AMI). Circulation 106, 3009-3017 (2002).

29. Stamm, C. et al. Autologous bone-marrow stem-cell transplantation for myocardial regeneration. Lancet 361, 45-46 (2003)

30. Wright, D.E., Wagers, A.J., Gulati, A.P., Johnson, F.L. \& Weissman, I.L. Physiological migration of hematopoietic stem and progenitor cells. Science 294 1933-1936 (2001).

31. Bryder, D. \& Jacobsen, S.E. Interleukin-3 supports expansion of long-term multilineage repopulating activity after multiple stem cell divisions in vitro. Blood $\mathbf{9 6}$, $1748-1755$ (2000)

32. Okabe, M., Ikawa, M., Kominami, K., Nakanishi, T. \& Nishimune, Y. 'Green mice' as a source of ubiquitous green cells. FEBS Lett. 407, 313-319 (1997).

33. Osawa, M., Hanada, K., Hamada, H. \& Nakauchi, H. Long-term Iymphohematopoietic reconstitution by a single CD34 lo/neg hematopoietic stem cell. Science $\mathbf{2 7 3}$ 242-245 (1996).

34. Kronenwett, R., Martin, S. \& Haas, R. The role of cytokines and adhesion molecules for mobilization of peripheral blood stem cells. Stem Cells 18, 320-330 (2000).

35. Bensinger, W.I. \& Storb, R. Allogenic peripheral blood stem cell transplantation. Rev. Clin. Exp. Hematol. 5, 67-86 (2001).
36. Zambrowicz, B.P. et al. Disruption of overlapping transcripts in the ROSA beta geo 26 gene trap strain leads to widespread expression of $\beta$-galactosidase in mouse embryos and hematopoietic cells. Proc. Natl. Acad. Sci. USA 94, 3789-3794 (1997).

37. Fleischmann, M. et al. Cardiac specific expression of the green fluorescent protein during early murine embryonic development. FEBS Lett. 440, 370-376 (1998).

38. Roell, W. et al. Cellular cardiomyoplasty improves survival after myocardial injury. Circulation 105, 2435-2441 (2002).

39. Agbulut, 0. et al. Temporal patterns of bone marrow cell differentiation following transplantation in doxorubicin-induced cardiomyopathy. Cardiovasc. Res. 58, 451-459 (2003).

40. Oh, H. et al. Cardiac progenitor cells from adult myocardium: homing, differentiation, and fusion after infarction. Proc. Natl. Acad. Sci. USA 100, 12313-12318 (2003).

41. Kang, H.-J. et al. Effects of intracoronary infusion of peripheral blood stem-cells mobilised with granulocyte-colony stimulating factor on left ventricular systolic function and restenosis after coronary stenting in myocardial infarction: the MAGIC cell randomised clinical trial. Lancet 363, 751-756 (2004).

42. Matsubara, H. Risk to the coronary arteries of intracoronary stem cell infusion and G-CSF cytokine therapy. Lancet 363, 746-747 (2004).

43. Shintani, S. et al. Mobilization of endothelial progenitor cells in patients with acute myocardial infarction. Circulation 103, 2776-2779 (2001).

44. Balsam, L.B. et al. Haematopoietic stem cells adopt mature haematopoietic fates in ischaemic myocardium. Nature 428, 664-668 (2004).

45. Murry, C.E. et al. Haematopoietic stem cells do not transdifferentiate into cardiac myocytes in myocardial infarcts. Nature 428, 668-673 (2004).

46. Roell, W. et al. Cellular cardiomyoplasty in a transgenic mouse model. Transplantation 73, 462-465 (2002).

47. Borge, O.J., Adolfsson, J. \& Jacobsen, A.M. Lymphoid-restricted development from multipotent candidate murine stem cells: distinct and complimentary functions of the c-kit and flt3- ligands. Blood 94, 3781-3790 (1999). 